\title{
Projet de loi sur les soins intégrés: démocratie directe et Médecins de famille Suisse
}

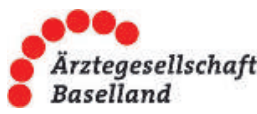

Correspondance: Dr Tobias Eichenberger Président Ärztegesellschaft Baselland Hammerstrasse 35 CH-4410 Liestal
Chères et chers Collègues,

Le 3 novembre dernier, trois quarts des 42 délégués de Médecins de famille Suisse ont pris la décision de ne pas soutenir le référendum contre le projet de loi sur le Managed care, porté par la FMH et plusieurs organisations de médecins, et de participer à un comité de soutien au projet de loi. Cette décision appelle un commentaire de notre part.

A la mi-septembre, donc avant la fin de la votation générale et la publication du résultat, la société de médecine de Bâle-Campagne et les trois autres sociétés cantonales de médecine à l'origine de la votation générale ainsi que la fmCh ont rédigé une décision de réserve commune, avec la teneur suivante: en cas de non (aussi minime soit-il), nous accepterons le résultat, féliciterons la FMH, soutiendrons sa ligne de conduite dans cette affaire et, surtout, n'adhérerons à aucun comité référendaire. Motifs: 1 . L'unité du corps médical et la cohésion de la FMH face à l'extérieur doivent passer avant les intérêts d'une organisation ou d'un groupe de sociétés dans une affaire particulière. 2. Il serait absurde de demander une votation générale pour ensuite ne pas tenir compte du résultat.

Depuis, le résultat a montré que toutes les structures dirigeantes de la FMH (président, Comité central, Chambre médicale et la plupart des comités des sociétés cantonales de médecine/CCM), à l'exception des initiants, avaient mal évalué la position de la base sur cette question. Cependant, le fait que ces mêmes personnes et organisations ont ensuite accepté sans réserve la décision de la base, et que les délégués de la
Le même résultat a été obtenu auprès des Médecins de famille de Genève. Nous ne connaissons du reste aucune organisation dont les membres auraient accepté le projet de loi lors d'une consultation. Si l'analyse minutieuse de nos spécialistes (en médecine de famille) de Bâle-Ville et Bâle-Campagne s'avère exacte, le projet de loi présente des risques, notamment parce qu'il ne précise aucunement que le pilotage incombe aux médecins de famille, ce qui remet en question le renforcement tant espéré de la médecine de famille. En fin de compte, avec ce projet, le Parlement a pour objectif d'aménager un budget global.

Bien trop souvent des fossés se creusent entre médecins de famille et spécialistes; une de nos tâches principales consiste, sur le plan cantonal, à les combler, à jeter des ponts et à améliorer la compréhension entre les différents groupes d'intérêts. Mais pour une fois, aucun fossé ne sépare les médecins de famille et les spécialistes: la méfiance à l'égard du projet de loi sur les soins intégrés et du Parlement, de même que la résistance face à ce projet, se retrouve dans toutes les spécialités, toutes les générations et tous les cantons.

Les tentatives désespérées des politiciens en vue de diffamer les opposants au projet, de les faire passer pour des sempiternels passéistes, opposés à toute réforme et réticents aux innovations, de qualifier leurs alliances de «malsaines» et de prédire une scission au sein du corps médical, montrent combien le Parlement a peur d'affronter un corps médical certes pluriel, mais uni face à l'extérieur et proche des patients et donc des électeurs, qui démontre sa capacité référendaire et

\section{«Mais pour une fois, aucun fossé ne sépare les médecins de famille et les spécialistes.»}

Chambre médicale du 26 octobre ont (certainement à contrecœur pour certains) jeté les bases de la campagne référendaire avec, à chaque fois, une large majorité, témoigne d'un grand professionnalisme et d'une conception intacte de ce qu'est et doit être la démocratie.

Chez nous, trois quarts des membres de la société des médecins de famille de Bâle-Ville et Bâle-Campagne se sont récemment prononcés en faveur du référendum, rejetant ainsi le projet de loi de manière encore plus nette que lors de la votation générale de la FMH. qui compte faire entendre sa voix en politique de santé. Nous savons tous combien nos médecins sont flexibles et innovants, eux qui doivent constamment s'adapter à des défis et à des conditions-cadres en perpétuelle évolution et qui sont à l'avant-garde dans bien des domaines (mise sur pied de réseaux, eHealth).

Et voilà qu'à présent, trois quarts des délégués de Médecins de famille Suisse déclarent mieux savoir que les autres. Sans tenir compte de l'interprétation erronée de l'ensemble des organes directeurs, sans 
légitimation démocratique et sans solidarité avec la FMH, ils ont décidé de s'opposer au référendum et de défendre un projet qui semble bien peu favorable au corps médical (même potentiellement dangereux pour les médecins de famille, selon nous) et qu'ils
Il viendra un temps où nous aurons besoin de la solidarité de tous les membres de la FMH pour défendre les intérêts réels des médecins de famille et nous serions alors bien mal inspirés de croire que nous serons plus forts seuls. Le peuple va accepter le référendum,

\section{«Nous ne connaissons du reste aucune organisation dont les membres auraient accepté le projet de loi lors d'une consultation.»}

estiment plus important que le poids politique de notre unité. Pour nous, cet entêtement idéologique sans fondement est un désastre tactique et politique. et les médecins de famille doivent compter parmi les gagnants!

Pour les représentants des médecins de famille auprès du Comité de la Société de médecine du canton de Bâle-Campagne:

Dr Beat Biedermann, médecine interne et oncologie FMH, Ergolzstrasse 31, 4414 Füllinsdorf

Dr Urs Buess, médecine générale FMH, Mühlemattstrasse 39, 4414 Füllinsdorf

Dr Aurelio Corfù, pédiatrie FMH, Käppelibodenweg 44, 4132 Muttenz

Dr Walter Frommenwiler, médecine générale et chirurgie FMH, Bahnhofstrasse 20, 4402 Frenkendorf

Dr Jürg Jutzi, médecine interne FMH, Gewerbestrasse 3, 4416 Bubendorf

Dr Thomas Reichenstein, médecine interne FMH, Poststrasse 2, 4302 Augst

Dr Martin Schwab, médecine générale FMH, Hauptstrasse 39, 4450 Sissach

Dr Angelika Senst, médecine générale FMH, Trottenstrasse 9, 4402 Frenkendorf

Dr Lukas Wagner, médecine générale FMH, Hauptstrasse 35, 4127 Birsfelden

Pour la présidence de la Société de médecine du canton de Bâle-Campagne:

Dr Tobias Eichenberger, président, urologie FMH, Hammerstrasse 35, 4410 Liestal 